\title{
ARTICLE OPEN \\ Impact of predictive medicine on therapeutic decision making: a randomized controlled trial in congenital heart disease
}

\author{
Huseyin Naci ${ }^{1}$, Maximilian Salcher-Konrad ${ }^{1}$, Alistair Mcguire ${ }^{1}$, Felix Berger ${ }^{2,3,4}$, Titus Kuehne ${ }^{2,4,5}$, Leonid Goubergrits ${ }^{5}$, \\ Vivek Muthurangu ${ }^{6}$, Ben Wilson ${ }^{7,8}$ and Marcus Kelm ${ }^{2,3,5}$
}

Computational modelling has made significant progress towards clinical application in recent years. In addition to providing detailed diagnostic data, these methods have the potential to simulate patient-specific interventions and to predict their outcome. Our objective was to evaluate to which extent patient-specific modelling influences treatment decisions in coarctation of the aorta $(\mathrm{CoA})$, a common congenital heart disease. We selected three cases with $\mathrm{CoA}$, two of which had borderline indications for intervention according to current clinical guidelines. The third case was not indicated for intervention according to guidelines. For each case, we generated two separate datasets. First dataset included conventional diagnostic parameters (echocardiography and magnetic resonance imaging). In the second, we added modelled parameters (pressure fields). For the two cases with borderline indications for intervention, the second dataset also included pressure fields after virtual stenting simulations. All parameters were computed by modelling methods that were previously validated. In an online-administered, invitation-only survey, we randomized 178 paediatric cardiologists to view either conventional (control) or add-on modelling (experimental) datasets. Primary endpoint was the proportion of participants recommending different therapeutic options: (1) surgery or catheter lab (collectively, "intervention") or (2) no intervention (follow-up with or without medication). Availability of data from computational predictive modelling influenced therapeutic decision making in two of three cases. There was a statistically significant association between group assignment and the recommendation of an intervention for one borderline case and one non-borderline case: $94.3 \%$ vs. 72.2\% (RR: $1.31,95 \% \mathrm{Cl}: 1.14-1.50, p=0.00$ ) and $18.8 \%$ vs. $5.1 \%$ (RR: $3.09,95 \% \mathrm{Cl}: 1.17-8.18, p=0.01$ ) of participants in the experimental and control groups respectively recommended an intervention. For the remaining case, there was no difference between the experimental and control group and the majority of participants recommended intervention. In sub-group analyses, findings were not affected by the experience level of participating cardiologists. Despite existing clinical guidelines, the therapy recommendations of the participating physicians were heterogeneous. Validated patient-specific computational modelling has the potential to influence treatment decisions. Future studies in broader areas are needed to evaluate whether differences in decisions result in improved outcomes (Trial Registration: NCT02700737).

npj Digital Medicine (2019)2:17; https://doi.org/10.1038/s41746-019-0085-1

\section{INTRODUCTION}

Health systems are increasingly aiming to adopt precision medicine to better account for the well-characterized heterogeneity among patient populations. ${ }^{1}$ In line with these goals, diagnostic methods, such as imaging, have improved substantially over the past years and are able to characterize patients at an ever increasing granularity. At the same time, recommendations in clinical practice guidelines led to major advances in evidencebased medicine. ${ }^{2}$ However, such guidelines are often inadequate to facilitate patient-centred care as they primarily aim to standardize treatment and therefore account only partially for inter-individual variability. ${ }^{3}$ This often leaves clinicians with significant uncertainty when choosing the optimal treatment strategy.

In coarctation of the aorta ( $\mathrm{CoA})$, a relatively common congenital heart disease, decisions on the timing and type of treatment (wait vs. pharmacological treatment vs. intervention) are crucial to prevent long-term sequalae such as persistent arterial hypertension and end-organ damage. ${ }^{4}$ However, recommendations do not always agree across different guidelines and thus leave room for debate, especially in clinically borderline cases. For example, the American College of Cardiology and the American Heart Association recommend intervention for primary $\mathrm{CoA}$ or restenosis if invasive peak-to-peak pressure gradients across the CoA exceed $20 \mathrm{mmHg}$ with or without significant narrowing. ${ }^{5}$ On the other hand, the European Society of Cardiology recommends interventional treatment if pressure gradients are higher than $20 \mathrm{mmHg}$ based on cuff pressures between upper and lower limbs in the presence of arterial hypertension. ${ }^{6}$

Patient-specific modelling has the potential to support individual therapy decisions and improve the success rate of

\footnotetext{
${ }^{1}$ LSE Health, Department of Health Policy, London School of Economics and Political Science, London, UK; ${ }^{2}$ German Heart Institute Berlin (DHZB), Berlin, Germany; ${ }^{3}$ Charité Universitätsmedizin Berlin, Pediatric Cardiology, Berlin, Germany; ${ }^{4}$ DZHK (German Centre for Cardiovascular Research), partner site Berlin, Berlin, Germany; ${ }^{5}$ Institute for Computational and Imaging Science in Cardiovascular Medicine, Charité - Universitätsmedizin Berlin, corporate member of Freie Universität Berlin, Humboldt-Universität zu

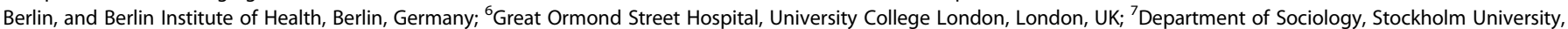
Stockholm, Sweden and ${ }^{8}$ Department of Methodology, London School of Economics and Political Science, London, UK

Correspondence: Huseyin Naci (h.naci@lse.ac.uk)
}

Received: 24 October 2018 Accepted: 1 February 2019

Published online: 19 March 2019 
interventions. For the evaluation of CoA, computational fluid dynamic (CFD) methods are of particular interest as they provide valuable information about hemodynamics such as pressure fields and flow profiles. ${ }^{7,8}$ In addition, they can be coupled with virtual intervention tools such as stenting of the CoA or aortic valve replacement which allow to predict the immediate hemodynamic effects of a given intervention. ${ }^{9,10}$ Therefore, such models can provide diagnostic as well as prognostic information concerning the hemodynamics before or after the simulated intervention. In turn, availability of patient-specific modelling data can reduce two key sources of uncertainty for clinical decision-making-diagnosis and prognosis-potentially influencing treatment recommendations. ${ }^{11}$

Despite recent advances of such patient-specific computational modelling concepts, the potential for clinical translation has not been subject to extensive evaluation. In recent years, there have been repeated calls to evaluate the effectiveness and costeffectiveness of these approaches in larger populations. ${ }^{12,13}$ However, an important first step is to evaluate their potential impact on clinician behaviour and decision making. Without demonstrable changes in clinicians' treatment decisions as a result of improved information garnered through computational modelling, subsequent changes in patient outcomes are unlikely.

Our objective was to design and implement a randomized controlled trial to evaluate whether and to what extent patientspecific computational modelling influences clinical treatment decisions in a common congenital heart condition (CoA). To do so, we designed an experiment with different levels of data presented to cardiologists to assess whether viewing image-based, patientspecific modelling altered the recommended course of action in hypothetical treatment scenarios.

\section{RESULTS}

\section{Participant characteristics}

Figure 1 shows the flow of participants in the study. Of 2235 eligible clinicians invited to participate, 2039 did not respond; 15 did not meet the inclusion criteria (either participated in CARDIOPROOF or had no experience treating patients with coarctation of the aorta over the past 6 months); and three did not give consent. A total of 178 cardiologists participated; 90 were randomly allocated to receive patient-specific computational modelling data for the first case (experimental) and 88 received conventional imaging parameters (control). After completing the questionnaire for the first case, 6 participants left the study, leaving 172 participants for the second case. Another participant left the survey after completing the questionnaire for the second case. A total of 171 participants were randomized to the third case:

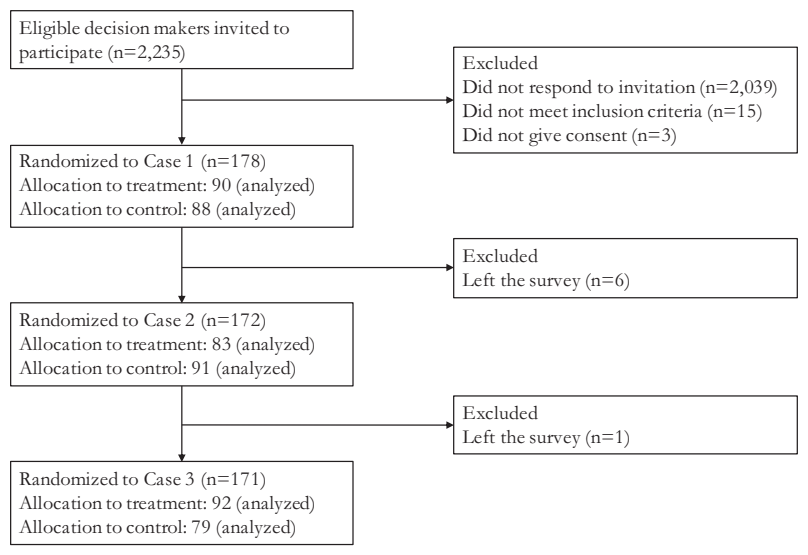

Fig. 1 Flow of participants in the trial
92 and 79 participants were randomized to experimental and control groups, respectively.

Participants in experimental and control arms of the trial had similar baseline characteristics. For the first two cases, there were no detectable differences between the groups (Table 1). There were only a few statistically significant between-group differences for the third case. For example, $87.3 \%$ of participants in the control group had over five years of experience with decision making in congenital heart disease as compared to $71.4 \%$ of those in the experimental group $(p=0.01)$. Similarly, $88.8 \%$ of the participants in the control group had treatment experience on more than five coarctation of the aorta cases in the past year as compared to $76.7 \%$ of those in the experimental group $(p=0.04)$.

\section{Outcomes}

Figure 2 shows the recommended course of action for each case. For the first case, overall, there was a statistically significant association between group assignment and recommended course of action $(p=0.00)$. For example, $70.5 \%$ of participants who were presented with patient-specific modelling data in addition to conventional parameters recommended referring the patient to the catheter lab as compared to $36.7 \%$ of participants who were given conventional parameters alone. Fewer participants in the experimental group recommended surgery compared to those in the control group $(23.9 \%$ vs. $35.6 \%$, respectively). In addition, fewer participants in the experimental group recommended no intervention (leave untreated and follow-up, 2.3\%; follow-up with medication, 2.4\%) compared to those in the control group (leave untreated and follow-up, 15.6\%; follow-up with medication, $12.2 \%)$. For the second case, there was no discernible difference between the groups in terms of their recommended course of action $(p=0.92)$. A similar proportion of participants recommended referring the patient to the catheter lab $(77.1 \%$ in treatment vs. $73.0 \%$ in control). A marginally statistically significant difference was observed between the two groups for the third case $(p=0.05)$.

Findings of the primary analysis are shown in Table 2. A higher proportion of participants recommended an intervention (either surgery or catheter lab) when presented with patient-specific modelling data in addition to conventional parameters for the first case (borderline) and third case (non-borderline). For the first case, 94.3\% of participants in the experimental group recommended an intervention as opposed to $72.2 \%$ of those in the control group (relative risk, RR: $1.31,95 \% \mathrm{Cl}: 1.14,1.50, p=0.00$ ); for the third case, $18.5 \%$ of participants in the experimental group and $5.1 \%$ of participants in the control group recommended an intervention (RR: $3.09,95 \% \mathrm{Cl}: 1.17,8.18, p=0.01$ ). There was no statistically significant difference between the participants who were and were not presented with patient-specific computational modelling data for the second case; $95.2 \%$ vs. $94.4 \%$ of those in the experimental group recommended an intervention (RR: 1.00, 95\% Cl: 0.94, 1.07, $p=0.82$ ).

Differences in the proportions of participants recommending either surgical intervention or catheter lab as opposed to no intervention (follow-up with or without medication) in experimental vs. control groups did not differ according to experience level (Table 3). For example, more participants recommended an intervention for the first case when they were presented with patient-specific modelling data in addition to conventional parameters compared to participants presented with only conventional imaging data, regardless of whether they had more than 5 years of experience in congenital heart disease (RR: 1.27, $95 \% \mathrm{Cl}: 1.09,1.49$ ) or less than 5 years of experience (RR: $1.47,95 \%$ $\mathrm{Cl}: 1.10,1.95)$. 
Table 1. Baseline characteristics of trial participants

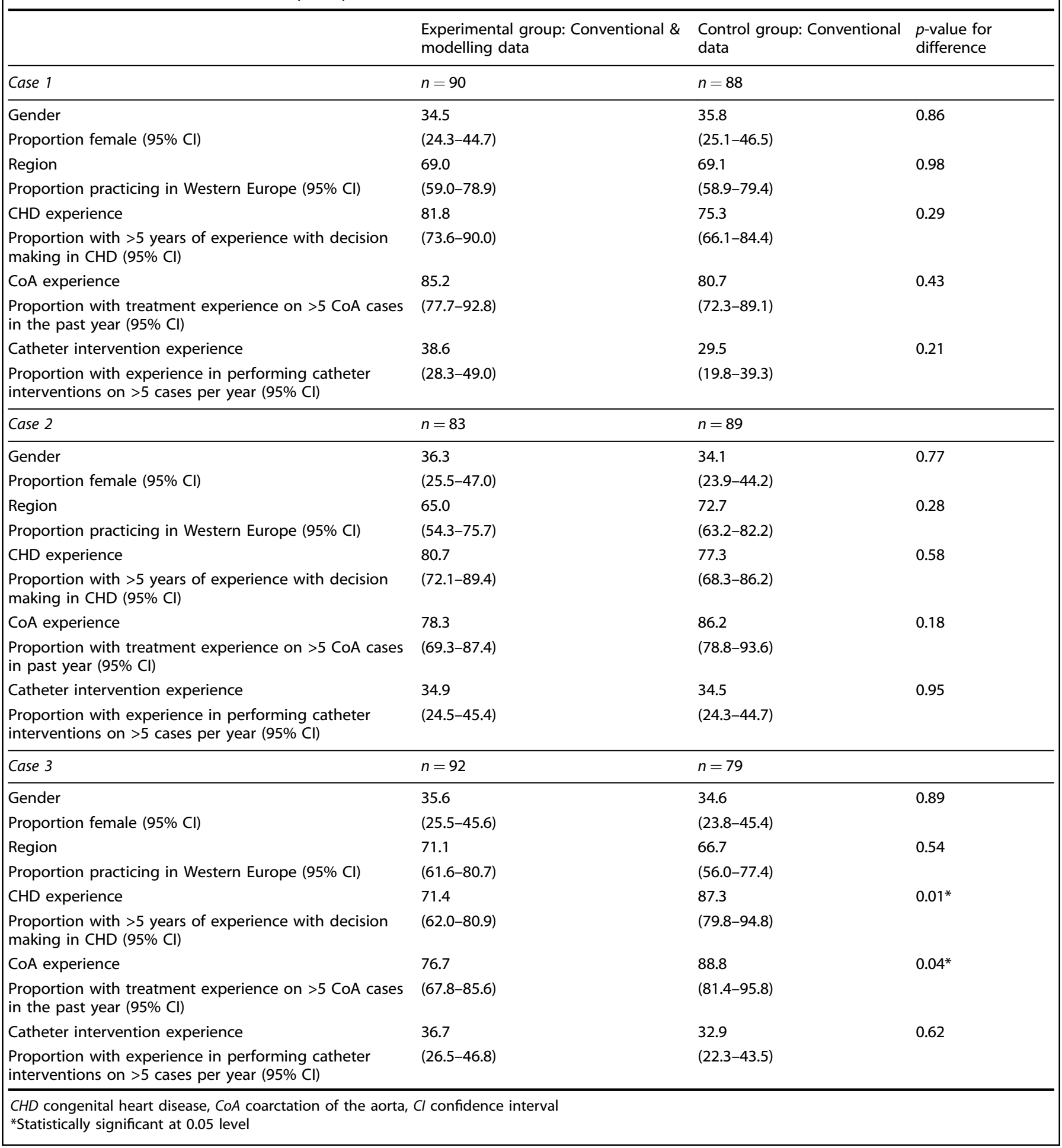

\section{DISCUSSION}

In this randomized controlled trial, we evaluated the treatment recommendations of physicians that were either based on current state-of-the-art diagnostic information or additional information from patient-specific computational models. We observed that despite existing clinical guidelines, the therapy recommendations of the participating physicians were quite heterogeneous for the individual patients when based on "conventional" diagnostic information. Patient-specific models appear to influence the recommended course of action. Cardiologists who were presented with computational modelling data in addition to conventional echocardiography and MRI data were more likely to recommend surgery or catheter intervention for two cases (one borderline and non-borderline). These findings were not affected by the experience level of the participating cardiologists.

Research on the development of integrated computer models of the mechanical, physical and biochemical functions of a living human body has drawn significant attention and has driven 


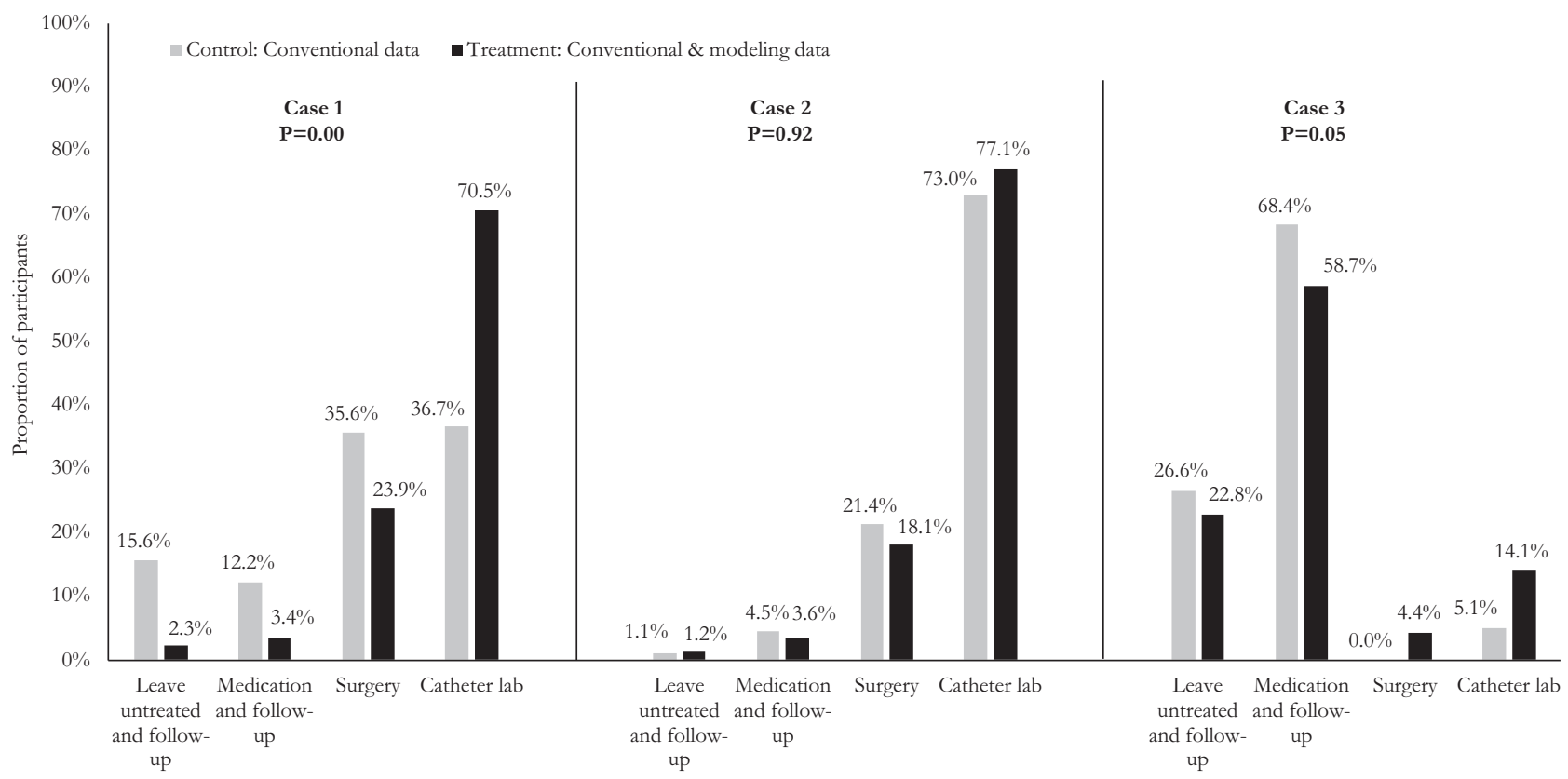

Fig. 2 Recommended course of action for each case. Experimental group includes participants randomized to see patient-specific modelling results in addition to conventional imaging data. Control group includes participants randomized to see only conventional imaging data. Fisher's exact test was used to statistically test for an association between group assignment and recommended course of action. $p$-value for case 1: 0.00; case 2: 0.92; and case 3: 0.05 . $p$-value $<0.05$ indicates a statistically significant difference between the groups

\begin{tabular}{|c|c|c|c|}
\hline & \multicolumn{2}{|c|}{ No. recommending intervention/total (\%) } & \multirow[b]{2}{*}{$\begin{array}{l}\text { Relative risk } \\
(95 \% \mathrm{Cl})\end{array}$} \\
\hline & $\begin{array}{l}\text { Experimental group } \\
\text { (Conventional \& } \\
\text { modelling data) }\end{array}$ & $\begin{array}{l}\text { Control group } \\
\text { (Conventional } \\
\text { data) }\end{array}$ & \\
\hline Case 1 & $83 / 88(94.3)$ & $65 / 90(72.2)$ & $1.31(1.14,1.50)$ \\
\hline Case 2 & 79/83 (95.2) & $84 / 89(94.4)$ & $1.00(0.94,1.07)$ \\
\hline Case 3 & 17/92 (18.5) & 4/79 (5.1) & $3.09(1.17,8.18)$ \\
\hline
\end{tabular}

investment for the past decade. ${ }^{14-16}$ This investment has been warranted on the premise that individualized risk prediction and virtual treatment planning have the potential to improve patient outcomes in many diseases. ${ }^{17}$ Indeed, previous models raised significant expectations to make decision support tools available for early diagnosis, disease prediction and outcome optimization. Recent studies increasingly provide supporting evidence on the clinical reliability and validity of models emanating from these research efforts. $^{18-21}$

The applied models in our study were validated in previous work. ${ }^{7,8,10}$ Uncertainties were addressed and it was shown that they can provide diagnostic information about pressure maps that are equivalent to invasive cardiac catheterization. ${ }^{22,23}$ In addition, it was shown that haemodynamic models after virtual treatment procedures can predict the hemodynamic effects of intervention. ${ }^{10}$ In the present study, for both cases that received stent placement there was good agreement between predicted pressure drop and pressure drop measured by catheter postintervention. However, investigated modelling methods are not meant to replace evidence-based guidelines that are based upon mid- or long-term outcome data. Instead, the modelling methods allow simulating the immediate hemodynamic effects of an intervention and thus may help answering whether a given intervention will provide hemodynamically meaningful results. This a-priori knowledge can be of clinical value, but such novel methods must be introduced into the clinical setting cautiously. It not only requires methods to be validated, but also to obtain knowledge about the impact of computational models on clinical decision-making process. This is what our trial starts to address.

Currently, little is known about how clinicians comprehend and utilize new patient-specific data, and whether this influences their treatment decisions. ${ }^{24-26}$ In this trial, we show that physicians who have access to patient-specific modeling data make different treatment recommendations. These findings are important to establish the decision impact of modelling data before investing in sizeable outcome trials in large patient populations. Future large trials should evaluate the appropriateness of making different treatment recommendations by mapping treatment decisions to mid-term and to long-term clinical outcomes. The design and findings of our scenario-based randomized experiment can offer insights for future evaluations of computational modelling approaches.

In accordance with what is widely known in clinical practice, we noted significant heterogeneity in decision making within each of the clinical cases. This result highlights the limitations of clinical guidelines in this area, despite their indisputable role in evidencebased medicine. In contrast, the use of the patient-specific models can result in distinctive shifts in decisions. This underscores the promises as well as the risks of computational models for personalized medicine. One can assume that it will be difficult for a user to deviate from the recommendation of a model-based decision support system if it predicts a given effect of an intervention. Advocates of patient-specific computational modelling often argue that individualizing treatment strategies would optimize the type and timing of interventions, which in turn reduce unnecessary or inappropriate invasive procedures, and help realize substantial cost savings. ${ }^{27,28}$

How did patient-specific modelling data influence treatment recommendations? We had anticipated that patient-specific modelling data would reduce uncertainty around diagnosis and prognosis, thereby resulting in different treatment 
Table 3. Proportion of participants recommending an intervention according to experience level

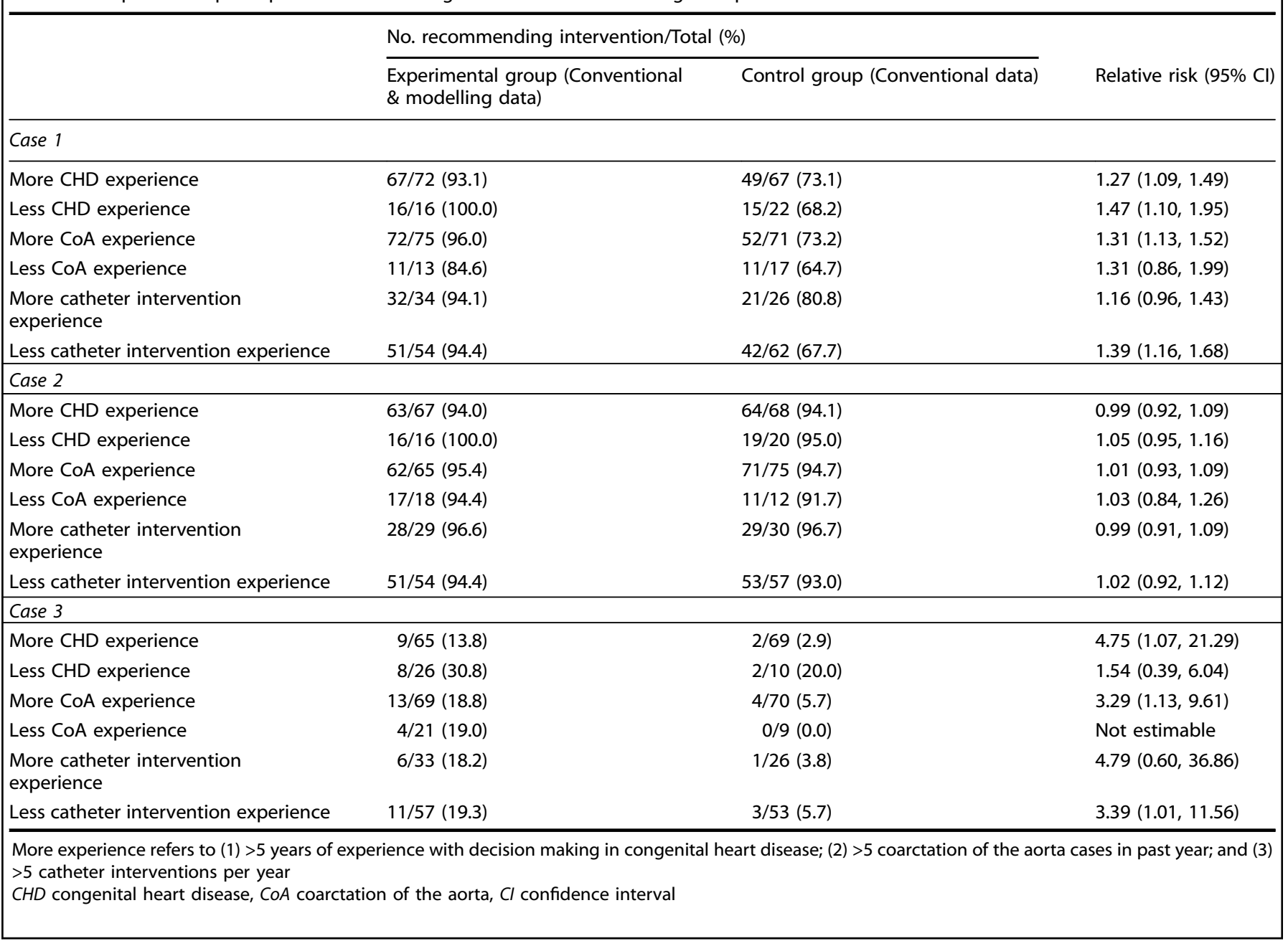

recommendations. In our trial, we presented computational modelling data on both the diagnosis and prognosis for two cases (Cases 1 and 2), and only on the diagnosis for one case (Case 3). Statistically significantly different recommendations were observed for Cases 1 and 3, indicating that it was reduced uncertainty-irrespective of its source-that was responsible for influencing treatment recommendations. In addition to the effect of reduced uncertainty, clinical decision making processes could be influenced by several other factors. These factors include participants' experience, hospital-specific practice, framing, search satisficing or even very subtle factors such as the scaling of the axes of graphs that have been extensively studied previously. ${ }^{29-31}$

In our study we have presented the participants primarily CFDbased models since hemodynamic parameters such as the distribution of pressure gradients are particularly important for CoA. The CFD models have also the advantage that the results are relatively easy to visualize for the user which is important for the straightforward implementation of the trial. In addition, the CFD models can also be used together with tools that allow performing virtual interventions. ${ }^{9}$ In the current study, we focused on virtual stent implantation to keep the experimental setup simple. The representation of surgical interventions is technically also possible, ${ }^{10}$ but would have increased the complexity of the trial set-up. In contrast, models that allow to assess the patient-specific response to pharmacological treatment are still under development and thus were also not used in our trial.
Providing the participants of our study with simulations obtained after virtual stenting for the first two cases may have primed them to consider the potential effects of stenting as opposed to other treatment alternatives. However, the proportion of participants who recommended referring patients to the catheter lab was not higher for Case 2 despite the availability of virtual stenting information. In addition, more participants recommended intervention for Case 3 despite no virtual stenting data presented for this case. These suggest that the priming effect of presenting virtual stenting data may be modest. Moreover, we cannot exclude that the presentation style of the preinterventional hemodynamic results could have influenced the participants. For Case 3, the graph of the pre-interventional hemodynamics shows a sharp pressure drop across the stenosis. In addition, the $x$-axes had a limited range which could have implied a relatively large pressure drop. These could be reasons why participants with model-based information (compared to participants provided only with conventional information) recommended an intervention in this case, despite the fact that the patient had no formal indication for intervention according to existing clinical guidelines. However, these explanations remain speculative. Therefore, future studies should be conducted that assess systematically how specific factors of modelling (including the presentation of modelling results) can shift decision in a given direction. 
The findings of our study should be interpreted in light of its limitations. We used data from three cases. Although a small number, these cases were carefully selected by experienced clinicians to demonstrate heterogeneity in clinical presentation and indication for intervention. Second, clearly this is an experimental study and we only considered hypothetical treatment decisions. It is conceivable that our study participants deviated from the types of decisions that they would have made in actual clinical settings. However, the extent to which their responses were affected by the hypothetical nature of our experiment should be similar in both the treatment and control groups, and therefore should not influence our findings. In addition, generalizability of our findings should be investigated further. Of 2235 cardiologists invited to take part in this study, 2039 did not respond. Another key limitation of our study is its sample. Cardiologists who agreed to participate represent a selected sample of potential users of patient-specific modelling approaches and may have a higher degree of knowledge and curiosity about modeling approaches. It is possible, therefore, participants of this trial had different attitudes towards innovative technologies (and hence treatment recommendations) than those who declined to take part.

In conclusion, our randomized experiment provides insights into the potential feasibility and clinical utility of novel modelling approaches aimed at individualizing treatment decisions for congenital heart conditions. This evaluation, despite focusing on a small number of cases, is nonetheless supportive of the use of individual patient computational modeling in arriving at a clinical decision. Whether observed differences in treatment decisions would ultimately improve patient outcomes must be investigated in future studies. Larger randomized controlled trials are therefore needed to test the impact of using novel modelling approaches in real-world clinical settings.

\section{METHODS}

Experimental and control groups

We designed a randomized trial focused on CoA to examine the treatment decision impact on practicing cardiologists with experience in treating this condition when they were presented with additional data generated by patient-specific computational modelling.

We generated two separate datasets for three actual clinical cases. The cases were selected by the clinical project partners in order to reflect clinical heterogeneity, amongst others with respect to age, vessel stenosis (location, degree and length) or presence of associated bicuspid aortic valve. In addition, the selected cases represented differing levels of borderline indication for intervention. Clinical details of the cases are shown in Fig. 3.

We created two datasets for each case. In the first dataset (control), we included conventional parameters (echocardiography and magnetic resonance imaging, MRI) currently recommended as standard diagnostic work-up in clinical practice guidelines (see Table 4). In the second dataset (experimental), in addition to the parameters in the first dataset, we included parameters obtained from clinically validated ${ }^{7,8}$ CFD-based patient-specific modelling. The additional parameters included information about anatomy (geometry, vessel diameter and radius along the centre line) and function (aortic flow field and pressure drop along the centre line). The anatomy of the aorta was segmented using ZIB-Amira (Zuse Institute Berlin, Germany). CFD simulations were based on fourdimensional phase contrast MRI data using Fluent (Version 14.5, ANSYS Inc., Canonsburg, PA, USA). A non-Newtonian blood model was used based on an adapted power law, while turbulence was accounted for by a k-w SST turbulence model. For spatial discretization, high quality unstructured volume meshes were generated producing approximately one million cells for each patient. Results from flow simulations were validated against catheter measured pressure drop and flow fields measured by fourdimensional phase contrast MRI.

We also presented virtual stenting data for the cases that had borderline indication for intervention. Virtual stenting was performed using a previously introduced interactive tool. ${ }^{9}$ Modelling parameters were presented for the pre-interventional state and a virtual post-
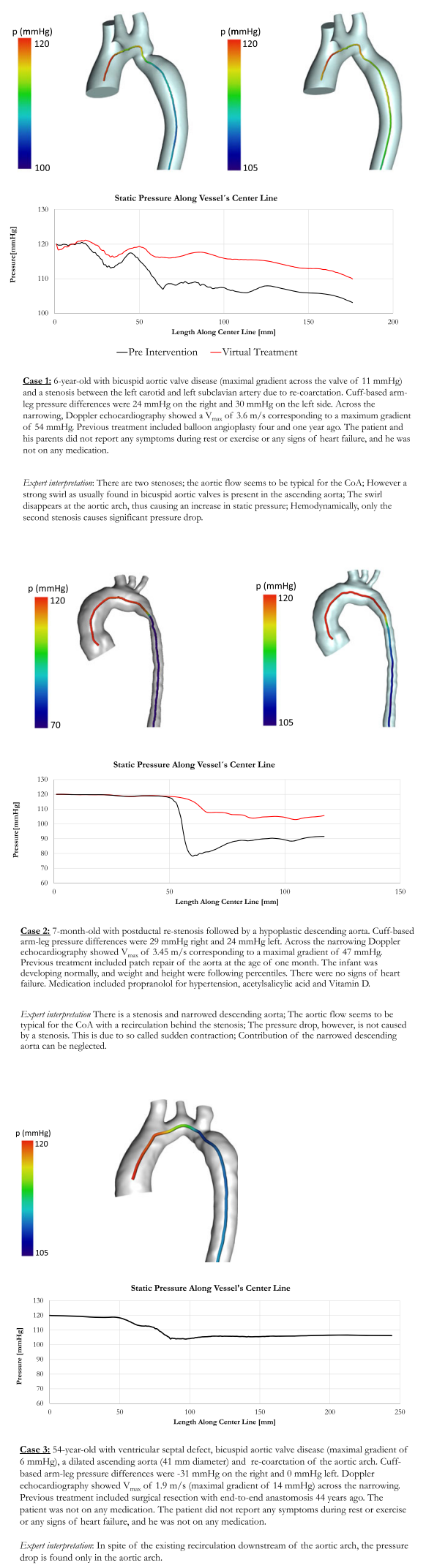

Fig. 3 Detailed presentation of cases

interventional state (stenting of the aorta). In addition, applied models were verified to correctly predict pressure drop post-intervention.

There was good agreement between modelled and invasive catheterization after stent placement for the two cases for which intervention was 
Table 4. List of data parameters

Panel A: Information provided to all participants:

- Clinical history

- Current problems

- Medication

- Patient characteristics/physical exam:

O Age

Weight

O Height

- Arterial blood pressure

- Any signs of heart failure

- Exercise capacity

Panel B: Control group

Conventional parameters

Echocardiography:

- Peak velocity across CoA (m/s)

- Pressure gradient at $\mathrm{CoA}(\mathrm{mmHg})$

- Left Ventricle (LV):

End-diastolic diameter (LVEDD in $\mathrm{mm}$ )

- Aortic valve: regurgitation

(degree), peak velocity $(\mathrm{m} / \mathrm{s})$

MRI:

$\cdot \mathrm{LV}$ :

End-diastolic volume (EDVin $\mathrm{mL} / \mathrm{m}^{2}$ )

End-systolic volume enlarged (ESV in $\mathrm{mL} / \mathrm{m}^{2}$ )

$O$ Ejection fraction (EF in \%)

O Dimensions:

Ascending aorta (mm)

Aortic arch (mm)

Aortic isthmus ( $\mathrm{mm}$ )

Descending aorta $(\mathrm{mm})$

Panel A shows the information provided to all participants. Panel B shows the list of conventional parameters provided to participants in the control group. Panel $C$ shows the list of conventional+patient-specific computational modelling parameters provided to participants in the experimental group

indicated according to guidelines (10 vs $12 \mathrm{mmHg}$ and 12 vs. $11 \mathrm{mmHg}$ ). The implanted stents did not vary in location, length or diameter compared to the virtual stents used within the simulations shown to the participants (Fig. 4). The remaining case did not receive any intervention, as formal guideline-based treatment indication was not fulfilled. Only modelling information, but no clinical post-treatment images, were presented to the participants for this case.

Clinicians who agreed to participate in the study received information about patient demographic characteristics (age, weight, height), history, symptoms, and clinical status (arterial blood pressure for all four extremities, signs of heart failure, and exercise capacity) for each of the three cases (see Supplementary Information for details). Whether study participants received the first or second dataset was dependent on their group allocation, as described below, as is the recruitment of participants.

\section{Trial design}

The randomized controlled trial design was used to minimise the potential threats to the internal validity of the study. We used a web-based survey

\section{A}

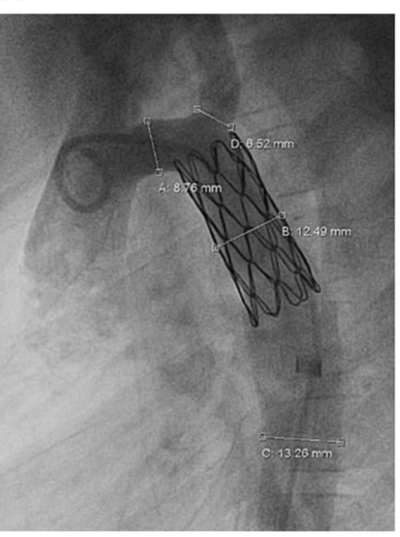

B

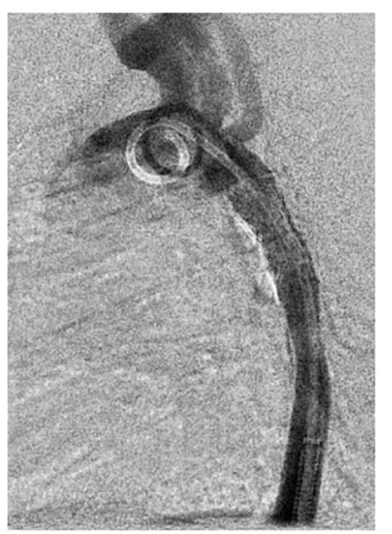

Fig. 4 Stent implantation in Cases 1 (a) and 2 (b)

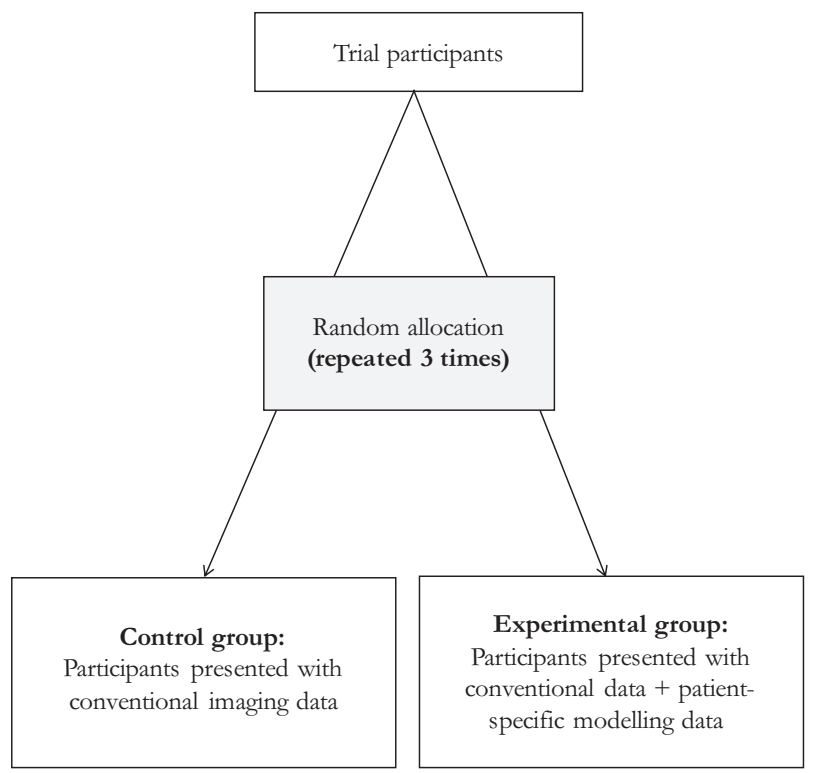

Fig. 5 Randomized controlled trial design

platform (Qualtrics) to generate the random allocation sequence (qualtrics. com). Randomized allocation was therefore completed centrally and investigators could not foresee assignment. ${ }^{32,33}$ Given the nature of the intervention, participants were aware of experimental vs. control assignment. However, investigators remained unaware of group allocation until after trial completion.

Randomization was at the case-level and was therefore repeated three times for each participant (i.e., each participant completed three randomized trials by the end of the study). Thus, it was possible for a trial participant to be randomized to the experimental group for one case and the control group for another. It was also possible for a participant to be repeatedly randomized to the experimental or control group three times. This design ensured that most participants had an opportunity to view CFD simulations at least for some cases. Trial design is shown in Fig. 5.

The target sample size was 118 (59 in each group) on the basis of an anticipated (hypothesized) effect size of $25 \%$ difference between the two groups in terms of the primary endpoint. Given the lack of similar evaluations in the literature, our hypothesized effect size was not based on previous empirical studies. We anticipated that an estimated $40 \%$ of clinicians presented with the conventional set of parameters (control) as compared to an estimated $65 \%$ of clinicians presented with patientspecific modelling parameters (experimental) would decide to recommend either surgery or catheter lab as opposed to follow-up with or without medication, giving an estimated effect size of $25 \%$. 
Practicing congenital, pediatric or interventional cardiologists were eligible for inclusion in the study if they had treated patients with coarctation of the aorta during the past 6 months. Clinicians were not eligible to take part in the trial if they were affiliated with the project team running the experiment, (the CARDIOPROOF consortium), or if they had pilot-tested the questionnaire in its previous iterations. Pilot-testing was conducted by cardiologist members of the CARDIOPROOF consortium project and non-consortium members from partner institutions to ensure readability and interpretability of the case summaries and accompanying questions.

We considered participants to have more experience if they had $>5$ years of experience with decision making in congenital heart disease; $>5$ coarctation of the aorta cases in the past year; and $>5$ catheter interventions per year.

Our recruitment strategy had two key elements. First, we manually searched and identified a list of practicing congenital, paediatric and interventional cardiologists in centres with experience in congenital heart disease in Europe and North America. When then contacted them via email. We also obtained access to the member lists of prominent organizations including the Association for European Paediatric and Congenital Cardiology (AEPC). Second, we attended the AEPC Conference and invited eligible clinicians to participate in the experiment using laptops provided by the study team. To incentivize recruitment on site, participants were eligible to voluntarily enter a lottery to win a tablet device, and the CARDIOPROOF consortium made a donation of 10 Euros per participant to a charity organisation (Save the Children). We did not provide any other remuneration to study participants.

\section{Survey implementation}

For the survey, the platform Qualtrics was used to administer the webbased questionnaire. ${ }^{34}$ The first part of the questionnaire presented the clinicians with the study information sheet and asked for their informed consent to take part in the experiment. The second part included a series of questions about participants' experience level with decision making in congenital heart disease in general, and $\mathrm{CoA}$ in particular. Participants indicating that they had no experience with treating patients with CoA were excluded at this stage. Remaining participants were then presented with the three cases, and depending on their group allocation, had access to either the conventional parameters or patient-specific computational modelling parameters. Questions pertaining to study endpoints then followed (see below). At the end of the questionnaire, all participants were asked about their demographic characteristics and geographic region of practice. In total, participants were able to complete the study in approximately $20 \mathrm{~min}$.

Study questions were devised to explore participants' willingness to recommend intervention in the presented cases depending on the type of information presented to them (experimental vs. control). Participants were first asked for their recommended course of action. Available options were treating the patient with medication and follow-up, leaving the patient untreated and following up, referring the patient to the catheter lab, and referring the patient to surgery. The primary endpoint was "decision to intervene", referring to a clinician decision to recommend either surgery or catheter lab (collectively, "intervention") as opposed to follow-up with or without medication ("no intervention").

\section{Statistical analysis}

We first used descriptive statistics to compare the experimental and control groups at baseline. Differences between study groups in terms of outcomes were then evaluated using Fisher's exact test for proportions, and chi square analysis with categories adjusted to avoid cells with $<5$ expected values. Statistical significance was defined as a $P$ value of $<0.05$ assuming a two-tailed hypothesis. All analyzes were repeated for subgroups according to experience level of participants. Statistical analyzes were performed in STATA (version 14.2; STATA Corp LLC, College Station, TX, USA).

The study was conducted in compliance with the London School of Economics and Political Science Research Ethics Policy and Code of Research Conduct. Due to the hypothetical nature of treatment decisions made as part of this experiment, the study was exempt from ethics review from the London School of Economics and Political Science Research Ethics Committee through the self-certification pathway, and approved by LSE Health. Informed consent was obtained from all participants.

\section{DATA AVAILABILITY}

The datasets generated during the current study are available from the corresponding author on reasonable request.

\section{ACKNOWLEDGEMENTS}

Financial support for this study was provided by the European Commission's $7^{\text {th }}$ Research Framework Programme (FP7). The funding agreement ensured the authors' independence in designing the study, interpreting the data, writing, and publishing the report. This study was performed as part of Cardioproof, a proof-of-concept study funded by the European Commission under FP7.

\section{AUTHOR CONTRIBUTIONS}

H.N., A.M., V.M., M.K., M.S.K., and T.K. conceived the study. H.N. and M.S.K. designed the trial and implemented it with input from M.K., T.K., V.M., L.G. H.N. and M.S.K. analyzed the data with input from B.W. and A.M. H.N. wrote the first draft of the paper. All authors critically revised and contributed to subsequent drafts.

\section{ADDITIONAL INFORMATION}

Supplementary information accompanies the paper on the npj Digital Medicine website (https://doi.org/10.1038/s41746-019-0085-1).

Competing interests: The authors declare no competing interests.

Publisher's note: Springer Nature remains neutral with regard to jurisdictional claims in published maps and institutional affiliations.

\section{REFERENCES}

1. Jameson, J. L. \& Longo, D. L. Precision medicine - personalized, problematic, and promising. New Engl. J. Med. 372, 2229-2234 (2015).

2. Grimshaw, J. M. \& Russell, I. T. Effect of clinical guidelines on medical practice: a systematic review of rigorous evaluations. Lancet 342, 1317-1322 (1993).

3. Goldberger, J. J. \& Buxton, A. E. Personalized medicine vs guideline-based medicine. JAMA 309, 2559-2560 (2013).

4. Tanous, D., Benson, L. \& Horlick, E. Coarctation of the aorta: evaluation and management. Curr. Opin. Cardiol. 24, 509-515 (2009).

5. Warnes, C. A. et al. ACC/AHA 2008 guidelines for the management of adults with congenital heart disease: a report of the American College of Cardiology/American Heart Association Task Force on Practice Guidelines (Writing Committee to Develop Guidelines on the Management of Adults With Congenital Heart Disease). Developed in Collaboration With the American Society of Echocardiography, Heart Rhythm Society, International Society for Adult Congenital Heart Disease, Society for Cardiovascular Angiography and Interventions, and Society of Thoracic Surgeons. J. Am. Coll. Cardiol. 52, e143-e263 (2008).

6. Baumgartner, H. et al. ESC Guidelines for the management of grown-up congenital heart disease (new version 2010). Eur. Heart J. 31, 2915-2957 (2010).

7. Goubergrits, L. et al. MRI-based computational fluid dynamics for diagnosis and treatment prediction: clinical validation study in patients with coarctation of aorta. J. Magn. Reson. Imaging 41, 909-916 (2015).

8. Goubergrits, L. et al. Is MRI-based CFD able to improve clinical treatment of coarctations of aorta? Ann. Biomed. Eng. 43, 168-176 (2015).

9. Neugebauer, M. et al. Interactive virtual stent planning for the treatment of coarctation of the aorta. Int. J. Comput. Assist. Radiol. Surg. 11, 133-144 (2016).

10. Kelm, M. et al. Model-based therapy planning allows prediction of haemodynamic outcome after aortic valve replacement. Sci. Rep. 7, 9897 (2017).

11. Han, P., Klein, W. \& Arora, N. Varieties of uncertainty in health care: a conceptual taxonomy. Med. Decis. Mak. 31, 828-838 (2011).

12. Biglino, G. et al. Computational modelling for congenital heart disease: how far are we from clinical translation?. Heart 103, 98-103 (2017).

13. Marsden, A. L. \& Feinstein, J. A. Computational modeling and engineering in pediatric and congenital heart disease. Curr. Opin. Pediatr. 27, 587 (2015).

14. Hunter, P. et al. A vision and strategy for the virtual physiological human in 2010 and beyond. Philos. Trans. R. Soc. Lond. A: Math., Phys. Eng. Sci. 368, 2595-2614 (2010).

15. Hunter, P. et al. A vision and strategy for the virtual physiological human: 2012 update. Interface Focus 3, 20130004 (2013). 
16. Smith, N. et al. euHeart: personalized and integrated cardiac care using patientspecific cardiovascular modelling. Interface Focus 1, 349-364 (2011).

17. Kohl, P. \& Noble, D. Systems biology and the virtual physiological human. Mol. Syst. Biol. 5, 292 (2009).

18. Kim, H. et al. Patient-specific modeling of blood flow and pressure in human coronary arteries. Ann. Biomed. Eng. 38, 3195-3209 (2010).

19. Yang, W., Chan, F. P., Reddy, V. M., Marsden, A. L. \& Feinstein, J. A. Flow simulations and validation for the first cohort of patients undergoing the Y-graft Fontan procedure. J. Thorac. Cardiovasc. Surg. 149, 247-255 (2015).

20. Mirzaee, $\mathrm{H}$. et al. MRI-based computational hemodynamics in patients with aortic coarctation using the lattice Boltzmann methods: clinical validation study. $J$. Magn. Reson. Imaging 45, 139-146 (2017).

21. Goubergrits, L. et al. MRI-based computational fluid dynamics for diagnosis and treatment prediction: Clinical validation study in patients with coarctation of aorta. J. Magn. Reson. Imaging 41, 909-916 (2015).

22. Goubergrits, L. et al. Patient-specific requirements and clinical validation of MRIbased pressure mapping: A two-center study in patients with aortic coarctation. J. Magn. Reson. Imaging 49, 81-89 (2018).

23. Fernandes, J. F. et al. CMR-based and time-shift corrected pressure gradients provide good agreement to invasive measurements in aortic coarctation. JACC Cardiovasc. Imaging 11, 1725-1727 (2018).

24. Cebral, J. R. \& Meng, H. Counterpoint: Realizing the clinical utility of computational fluid dynamics-Closing the gap. Am. J. Neuroradiol. 33, 396-398 (2012).

25. Kallmes, D. F. Point: CFD—computational fluid dynamics or confounding factor dissemination. Am. J.Neuroradiol. 33, (395-396 (2012).

26. Vignon-Clementel, I. E., Marsden, A. L. \& Feinstein, J. A. A primer on computational simulation in congenital heart disease for the clinician. Prog. Pediatr. Cardiol. 30, 3-13 (2010).

27. Kim, H. J. et al. Patient-specific modeling of blood flow and pressure in human coronary arteries. Ann. Biomed. Eng. 38, 3195-3209 (2010).

28. Taylor, C. A. \& Steinman, D. A. Image-based modeling of blood flow and vessel wall dynamics: applications, methods and future directions. Ann. Biomed. Eng. 38, 1188-1203 (2010).
29. Gill, T. M. The central role of prognosis in clinical decision making. JAMA $\mathbf{3 0 7}$ 199-200 (2012).

30. Croskerry, P. From mindless to mindful practice-cognitive bias and clinical decision making. N. Engl. J. Med. 368, 2445-2448 (2013).

31. Cardoso, R. L., Leite, R. O. \& de Aquino, A. C. A graph is worth a thousand words: how overconfidence and graphical disclosure of numerical information influence financial analysts accuracy on decision making. PLOS ONE 11, e0160443 (2016).

32. Clark, L., Fairhurst, C. \& Torgerson, D. J. Allocation concealment in randomised controlled trials: are we getting better?. BMJ 355, i5663, https://doi.org/10.1136/ bmj.i5663 (2016).

33. Schulz, K. F. \& Grimes, D. A. Generation of allocation sequences in randomised trials: chance, not choice. Lancet 359, 515-519 (2002).

34. Snow, J. \& Mann, M. Qualtrics survey software: handbook for research professionals. Qualtrics Labs, Inc. 2013.

Open Access This article is licensed under a Creative Commons Attribution 4.0 International License, which permits use, sharing, adaptation, distribution and reproduction in any medium or format, as long as you give appropriate credit to the original author(s) and the source, provide a link to the Creative Commons license, and indicate if changes were made. The images or other third party material in this article are included in the article's Creative Commons license, unless indicated otherwise in a credit line to the material. If material is not included in the article's Creative Commons license and your intended use is not permitted by statutory regulation or exceeds the permitted use, you will need to obtain permission directly from the copyright holder. To view a copy of this license, visit http://creativecommons. org/licenses/by/4.0/.

(c) The Author(s) 2019 